\title{
Effect of intact oxaliplatin in plasma on a cold allodynia after multiple administrations in colorectal cancer model rats
}

\author{
Yukako Ito, Shinji Kobuchi, Mako Takeda, Miki Morimoto \\ Department of Pharmacokinetics, Kyoto Pharmaceutical University, Yamashina-ku, Kyoto, Japan \\ Contributions: (I) Conception and design: Y Ito; (II) Administrative support: Y Ito; (III) Provision of study materials or patients: None; (IV) Collection \\ and assembly of data: M Takeda, M Morimoto; (V) Data analysis and interpretation: S Kobuchi; (VI) Manuscript writing: All authors; (VII) Final \\ approval of manuscript: All authors. \\ Correspondence to: Yukako Ito, PhD. Department of Pharmacokinetics, Kyoto Pharmaceutical University, Yamashina-ku, Kyoto 607-8412, Japan. \\ Email: yukako@mb.kyoto-phu.ac.jp.
}

\begin{abstract}
Background: Oxaliplatin (L-OHP)-induced acute neuropathy is a major factor for influencing treatment compliance in patients receiving chemotherapy for colorectal cancer (CRC). Acute neuropathy is caused by the intact L-OHP affecting the function of transient receptor potential vanilloid 1 (TRPV1) channel. In this study, the effectiveness of the detection of the intact L-OHP and the association with the severity of L-OHP-induced acute neuropathy were investigated using a rat model of CRC.

Methods: Wistar male rats were prepared as models of CRC by using 1,2-dimethylhydrazine (DMH) and dextran sulfate. Intravenous L-OHP was administered (weekly) to CRC rats for 4 weeks, at doses of 3, 5, or $8 \mathrm{mg} / \mathrm{kg}$, respectively. Pharmacokinetic and tumor distribution profiles of animals with intact L-OHP were observed on days 1 and 22. Cold allodynia was determined as a read-out of acute neuropathy using the acetone test over the 4 weeks.

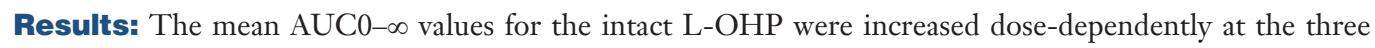
doses. The accumulation of intact L-OHP was confirmed following an increase in L-OHP concentration on day 22. Acute neuropathy was observed from day 2 at all doses and the withdrawal response correlated with $\operatorname{AUC}\left(\mathrm{R}^{2}=0.9816\right)$.

Conclusions: To prevent the onset of L-OHP-induced acute neuropathy, the timely detection of the intact L-OHP is important. These findings could be a basis of the establishment a pharmacokinetic and toxicodynamic model to aid the planning of therapy cycle completion for CRC.
\end{abstract}

Keywords: Oxaliplatin (L-OHP); acute neuropathy; pharmacokinetic; colorectal cancer model; rat

Submitted Mar 04, 2020. Accepted for publication Jun 30, 2020.

doi: 10.21037/apm-20-542

View this article at: http://dx.doi.org/10.21037/apm-20-542

\section{Introduction}

Oxaliplatin (L-OHP) in combination with fluoropyrimidine has emerged as the standard chemotherapy cocktail for colorectal cancer in both adjuvant and palliative settings (1-5). However, its neurotoxicity is a major limiting effect that often results in a dose reduction or early termination of the drug regimen. In addition, the long-term toxicity associated with the treatment may adversely affect a patient's quality of life for many years after the termination of treatment (6). This acute neurotoxicity usually occurs in the first round of therapy, making it a primary determining factor in continuing the therapy regimen. The mechanism of L-OHP action has been linked to the prolonged opening of calcium-dependent voltage-gated sodium channels induced by the chelation of calcium facilitated by oxalate (7-10). The prolonged opening of these sodium channels may induce cellular stress. In addition, the accumulation of platinum compounds in the dorsal root ganglion cells, may also result in damage to the cell body and axon (11-13). 


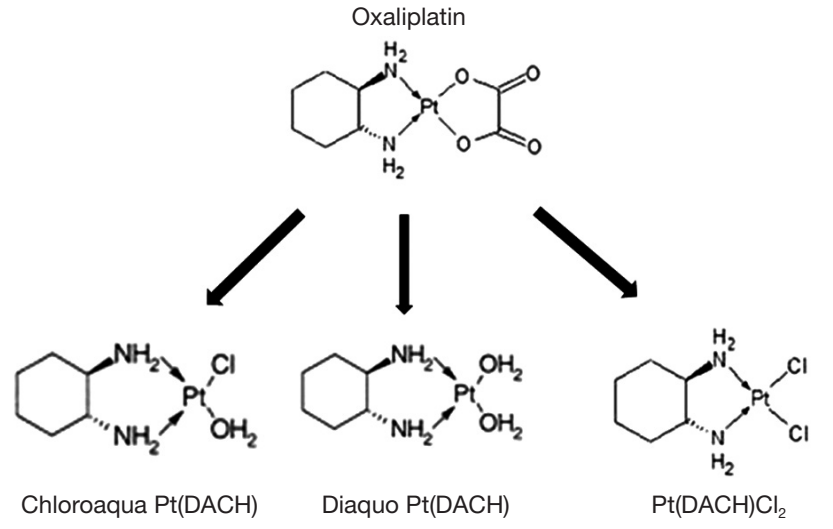

Figure 1 Biotransformation of oxaliplatin and pharmacological major products.

This damage may contribute to the development of the chronic peripheral sensory neurotoxicity of L-OHP. It has been suggested that L-OHP and/or its metabolites may increase calcium influx into the cytosol of peripheral neurons. This may enhance the activation of sodium and potassium channels, calcium regulated transcription factors, and intracellular signaling involving calcium-dependent protein kinase activity $(14,15)$. Once administered it has been suggested that L-OHP undergoes rapid non-enzymatic biotransformation reactions in the presence of water and nucleophiles, including chloride, resulting in the formation of reactive species including monochloro-, dichloro-, and diaquo-DACH (cyclohexane-1,2-diamine) platinum as shown in Figure 1 (16). In in vitro studies, degradation of L-OHP in the presence of chloride was reported to result in the formation of monochloro-monooxalato ringopened complex, $[\mathrm{Pt}(\mathrm{DACH}) \text { oxCl}]^{-}$(17). This complex can revert back into $\mathrm{L}-\mathrm{OHP}$ or transform into $\mathrm{Pt}(\mathrm{DACH}) \mathrm{Cl}_{2}$; thus, this complex biochemical pathway results in a very slow degradation cycle for L-OHP. The contribution of these different ligand displacement reactions to the in vivo clearance of L-OHP are not well understood. To date, there have been few studies focused on in vitro-in vivo extrapolation methods to predict their effects on the in vivo clearance of L-OHP. These in vitro and clinical datasets provide an opportunity to develop experimental approaches for the prediction of these effects on L-OHP clearance in vivo.

Han et al. reported that the degradation and in vivo clearance of L-OHP is associated with its neurotoxicity (18). Ito et al. reported a sensitive and selective method for detecting intact L-OHP using a hydrophilic column (19). This detection method has been used to establish the link between L-OHP and acute neuropathy. Therefore, the concentration of intact L-OHP might be a predictive factor for acute neurotoxicity. In this study, the association between the plasma concentration of intact L-OHP and the extent of acute neurotoxicity was evaluated in a rat model of colorectal cancer.

We present the following article in accordance with the ARRIVE reporting checklist (available at http://dx.doi. org/10.21037/apm-20-542).

\section{Methods}

\section{Materials}

Elplat $^{\circledR}$ (L-OHP) was purchased from Yakult Honsha Co., Ltd. While pure L-OHP was obtained from Wako Pure Chemical Industries Ltd. (Osaka, Japan). Male Wistar rats were obtained from Japan SLC Inc. (Hamamatsu, Japan). A standard solid-meal commercial diet (LabDiet; Nousan Corp., Yokohama, Japan) was used to feed all the animals. All other materials were of reagent grade and were used in the form they were received.

\section{Colorectal cancer model rats}

Colorectal tumors were induced in rats using the rapid induction method (20). Briefly, 10-week-old male Wistar rats were administered 1,2-dimethylhydrazine $(40 \mathrm{mg} / \mathrm{kg})$ once a week and were given drinking water containing $1.0 \%$ dextran sulfate sodium solution freely for 2 weeks. The rats were housed in a room with a 12-hour light/dark cycle, controlled temperature of $23 \pm 2{ }^{\circ} \mathrm{C}$, and humidity levels of $50 \% \pm 10 \%$. The animals were fed a moderate fat basal diet with free access to drinking water (Nippon SLC Co., Ltd., Hamamatsu, Japan). After 10 weeks, aberrant crypt foci (ACF) were identified using an endoscope (AVS AE-C1, OLYMPUS. Co. Ltd., Tokyo, Japan). The body temperature of the animals was maintained at $37{ }^{\circ} \mathrm{C}$ during the experiment.

\section{Pharmacokinetic study of intact L-OHP in colorectal cancer rats}

Intravenous injections were prepared by dissolving L-OHP $(100 \mathrm{mg})$ in $20 \mathrm{~mL}$ of saline. Male Wistar colorectal positive rats $(306 \pm 10 \mathrm{~g}$ in mass) were anesthetized using $2 \%$ isoflurane before weekly administration of intravenous L-OHP at $3.0,5.0$, or $8.0 \mathrm{mg} / \mathrm{kg}$ on days $0,7,14$, and 21 , 
according to our previous report related in L-OHP induced neuropathy (21). Each concentration represented a unique group with 4 to 6 rats per group. Blood was collected from the right jugular vein at 3, 5, 10, 20, 30, 45, 60, 90, and $120 \mathrm{~min}$ on days 0 and 21. Plasma was obtained by centrifuging the blood samples at 12,000 rpm for $15 \mathrm{~min}$ at $4{ }^{\circ} \mathrm{C}$ (KUBOTA 3700; Kubota Corp., Tokyo, Japan) and was stored at $-80^{\circ} \mathrm{C}$ until it could be assayed.

\section{Acetone test for cold allodynia}

Each rat was placed in a clear plastic box $(20 \mathrm{~cm} \times 20 \mathrm{~cm})$ and was allowed to get habituated to this environment for $30 \mathrm{~min}$ prior to testing. Fifty microliters of acetone (Wako Pure Chemical Industries, Ltd., Osaka) were sprayed onto the plantar skin of each hind paw, and the number of withdrawal responses was counted for $40 \mathrm{~s}$ from the start of the acetone spray. This design is in accordance with other previously described methods (22). That is, it is consisted with the three experimental group (L-OHP of 3, 5 and $8 \mathrm{mg} / \mathrm{kg}$ ) and the control group (saline). The test was performed 6 times (3 times per hind limb) per animal.

\section{Analysis of intact L-OHP}

L-OHP was analyzed by liquid chromatography/tandem mass spectrometry (LC/MS/MS), using the modified method of the Ito et al. (19). That is, the ultrafiltration was arranged to the sample centrifugation as the deproteinization procedure, because both of procedure is equivalent and centrifugation has low costs (23). Fresh standards were prepared for the quantification of L-OHP in plasma. Plasma $(100 \mu \mathrm{L}$ per sample, or $90 \mu \mathrm{L}$ of blank matrix plus $10 \mu \mathrm{L}$ of stock solution for standards) samples were mixed with acetonitrile $(200 \mu \mathrm{L})$ for deproteinization, according to our previous report (24). This was followed by vortexing and centrifugation at 13,000 $\mathrm{rpm}$ for $5 \mathrm{~min}$ (KUBOTA 3700; Kubota Corp., Tokyo, Japan); $100 \mu \mathrm{L}$ of the supernatant was then transferred into a vial, and $20 \mu \mathrm{L}$ was injected into the LC/MS/MS instrument. This procedure is same to the ultrafiltration of the sample by Ito et al. (19). The calibration range was 0.1 to $250 \mu \mathrm{g} / \mathrm{mL}$ for L-OHP in plasma. Chromatographic analysis was performed on a high-performance liquid chromatography system (SHIMADZU, Kyoto, Japan) coupled with an API 3200 mass spectrometer (Applied Biosystems/MDS Sciex, Foster City, CA, USA). The mobile phase consisted of methanol. The detection of L-OHP was achieved using an isocratic system, at a flow rate of $0.2 \mathrm{~mL} / \mathrm{min}$ and total run time of $3 \mathrm{~min}$. The MS instrument was operated in the positive ion mode. The multiple reaction monitoring transition of L-OHP was 639.0/491.0 (Q1/Q3).

\section{Statistics}

All values are expressed as their mean \pm SD. Statistical significance was evaluated by one-way analysis of variance (one-way ANOVA) with post-hoc comparisons by the Bonferroni test.

\section{Ethical statement}

All animal protocols were approved by the Institutional Animal care and Use Committee (No. PKPD-16-001) and were conducted in accordance with the Guidelines for Animal Experimentation, Kyoto Pharmaceutical University.

\section{Results}

\section{Pharmacokinetic profiles of L-OHP}

Plasma concentration profiles of intact L-OHP on days 1 and 22, and the pharmacokinetic parameters are shown in Figure 2 and Table 1 respectively. These values are reported as Pt levels, which include only intact L-OHP. The accumulation of L-OHP was observed at doses of 3,5 and $8 \mathrm{mg} / \mathrm{kg}$ on day 22 . The mean $\mathrm{AUC} 0-\infty$ values obtained on day 22 were significantly higher than those obtained on day $1(\mathrm{P}<0.05)$, at all three doses. Alternatively, the repeated administration reduced clearance of L-OHP. This is related to the longer retention of L-OHP in the circulation. Although our previous report was also studied that the lower plasma concentration and the localization of $\mathrm{L}-\mathrm{OHP}$ in colorectal tumor tissue were associated with the high intensity of the mechanical allodynia after the L-OHP administration (21), the intact L-OHP was not different distribution.

\section{L-OHP-induced cold allodynia}

Cold allodynia was evaluated using an acetone test performed over 28 days (Figure 3). On day 2, the withdrawal response of all three treated groups was higher than that of the control group. In the $8 \mathrm{mg} / \mathrm{kg}$ group, the number of responses remained significantly high against the control 

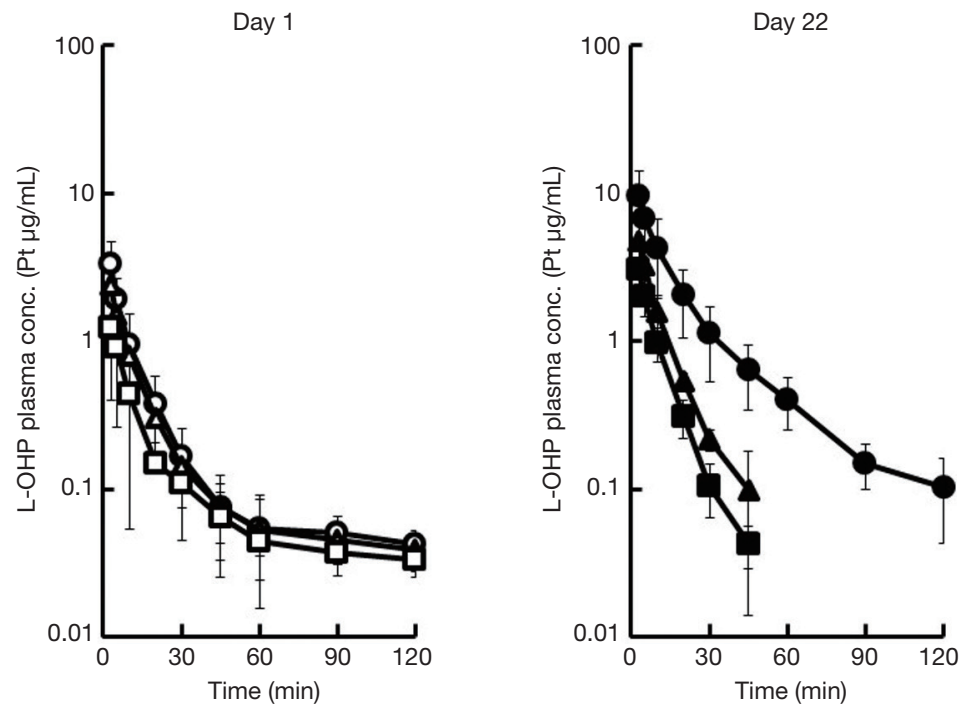

Figure 2 Pharmacokinetic profiles of L-OHP, on days 1 and 22 after intravenous administration at 3.0, 5.0, and 8.0 mg/kg to colorectal cancer rats. Open symbols represent day 1 and closed symbols represent day 22. The administered doses, 3.0, 5.0 and $8.0 \mathrm{mg} / \mathrm{kg}$, are represented as square, triangle, and circle symbols, respectively. Each point shows the mean \pm SD of four experiments. L-OHP, oxaliplatin; SD, standard deviation.

Table 1 Pharmacokinetic parameters of L-OHP

\begin{tabular}{|c|c|c|c|c|c|c|}
\hline $\begin{array}{l}\text { Pharmacokinetic } \\
\text { parameters }\end{array}$ & \multicolumn{2}{|c|}{$3 \mathrm{mg} / \mathrm{kg}$} & \multicolumn{2}{|c|}{$5 \mathrm{mg} / \mathrm{kg}$} & \multicolumn{2}{|c|}{$8 \mathrm{mg} / \mathrm{kg}$} \\
\hline t1/2 (h) & $1.1 \pm 0.3$ & $0.2 \pm 0.2^{*}$ & $0.6 \pm 0.4$ & $0.5 \pm 0.5$ & $1.0 \pm 0.4$ & $0.6 \pm 0.3$ \\
\hline CL (L/kg) & $3.8 \pm 1.0$ & $2.6 \pm 0.7^{*}$ & $4.4 \pm 1.7$ & $2.4 \pm 0.3^{\star}$ & $6.1 \pm 3.1$ & $1.6 \pm 0.7^{\star}$ \\
\hline $\operatorname{Vd}(\mathrm{L} / \mathrm{h} / \mathrm{kg})$ & $6.0 \pm 2.4$ & $1.0 \pm 0.8^{*}$ & $3.3 \pm 1.8$ & $1.9 \pm 1.9$ & $8.3 \pm 6.6$ & $1.4 \pm 1.2$ \\
\hline
\end{tabular}

Mean $\pm \mathrm{SD}(\mathrm{N}=4-8)$. * $\mathrm{P}<0.05$ significantly difference between days 1 and 22; \#, $\mathrm{P}<0.05$ significantly different from $3 \mathrm{mg} / \mathrm{kg}$. L-OHP, oxaliplatin; CL, clearance; Vd, volume of distribution; AUC, area under the curve; SD, standard deviation.

group over the course of the 28 days. Other groups showed a dose-dependent decrease in the withdrawal response. As cold allodynia is related to acute neuropathy, the onset of cold allodynia on day 2 suggested a correlation with acute neuropathy. The withdrawal response was maintained as a result of the continued administration of L-OHP. These results indicate that the sensitivity of L-OHPinduced neuropathy is related to the concentration of L-OHP (19). Therefore, we can assume that the multiple dose administration increased the incidence of L-OHP induced neuropathy. Therefore, the acute allodynia was predominated by not the $\mathrm{Pt}$ related compounds but the intact L-OHP.

\section{Correlation between pharmacokinetics and toxicodynamics of $L-O H P$}

In order to prevent the onset of cold allodynia, the association between the withdrawal response and the AUC value was investigated. The correlation between the withdrawal response of cold allodynia and the pharmacokinetic parameters of L-OHP was evaluated in Figure $4\left(\mathrm{R}^{2}=0.9816\right)$. There was a good correlation between these parameters. This suggests that monitoring of L-OHP in the plasma can potentially be used to predict the onset of cold allodynia, indicating that it would be possible to prevent acute neuropathy in patients by monitoring L-OHP levels in the serum and reducing the dosage accordingly. 


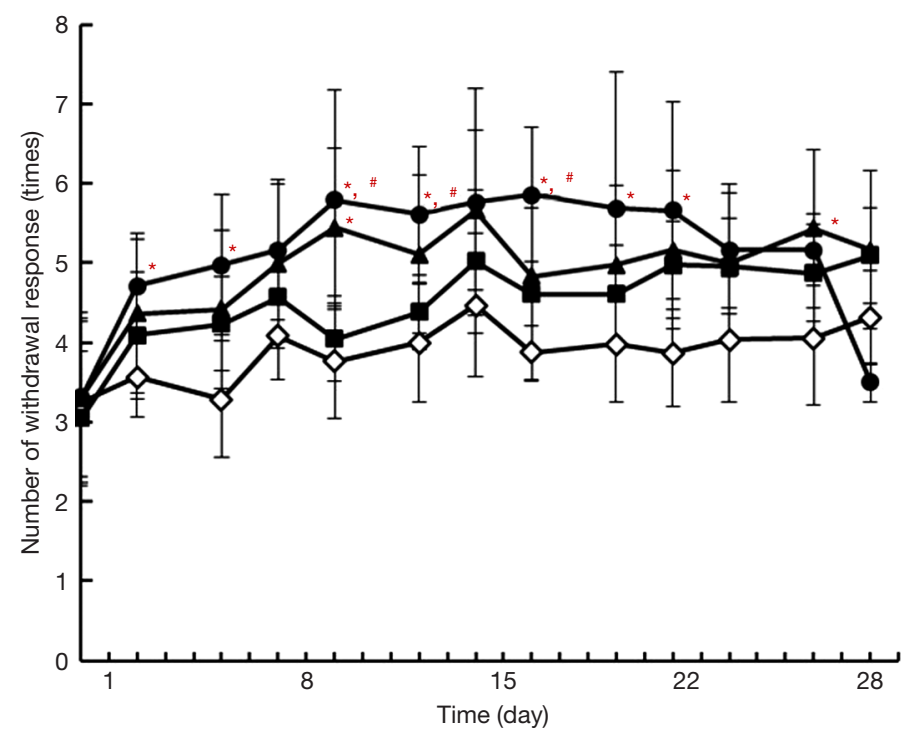

Figure 3 L-OHP-induced acute neuropathy in acetone test, evaluated as paw withdrawal thresholds in colorectal rats at the doses of 3.0 ( $\mathbf{})$, $5.0(\boldsymbol{\Delta})$, and $8.0(\bullet) \mathrm{mg} / \mathrm{kg}$, and control $(\diamond)$. Each point shows the mean $\pm \mathrm{SD}$ of four experiments. * $, \mathrm{P}<0.05, v s$. control; ${ }^{*}, \mathrm{P}<0.05, v s .3 \mathrm{mg} / \mathrm{kg}$ from one-way ANOVA post-hoc comparisons by the Bonferroni test. L-OHP, oxaliplatin; SD, standard deviation.

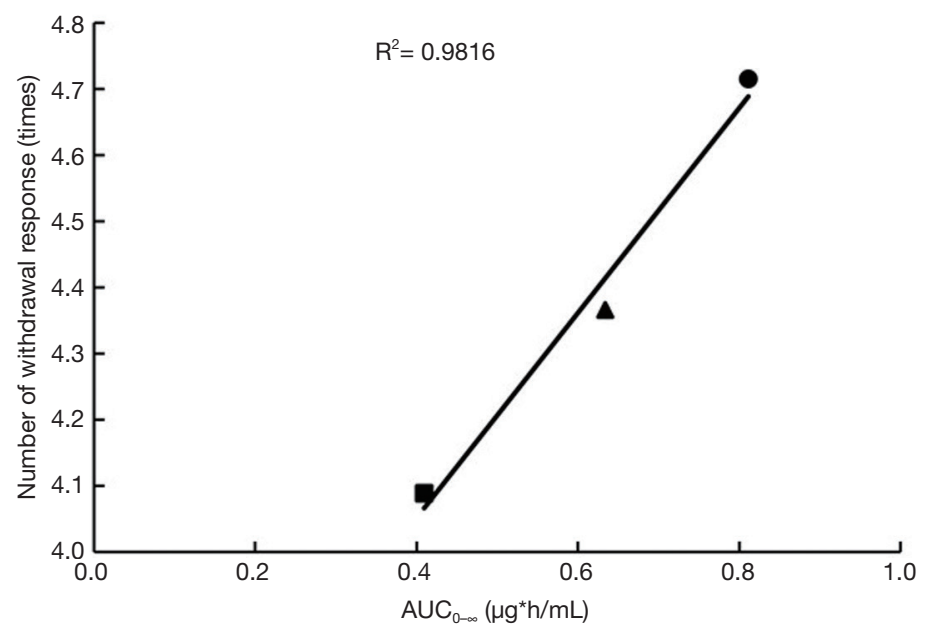

Figure 4 Correlation between the AUC of L-OHP and chronic neuropathy on day 21. evaluated as paw withdrawal thresholds in rats at the doses of $3.0(\bullet), 5.0(\boldsymbol{\Delta})$, and $8.0(\bullet) \mathrm{mg} / \mathrm{kg}$. Each point shows the mean \pm SD of four experiments. L-OHP, oxaliplatin; SD, standard deviation; AUC, area under the curve.

\section{Discussion}

L-OHP-induced acute neuropathy is among the first adverse effects experienced by patients being treated for colorectal cancer. As a result of this adverse effect, some patients may discontinue treatment, thereby reducing the efficacy of treatment. As a care strategy, a specialized glove was designed to protect patients against cold stimulation.
However, this strategy failed to prove effective $(25,26)$ Therefore, neuropathy is a serious factor in response to chemotherapy, and a factor worth consideration when studying colorectal cancer survival rates. In a previous study, we reported a correlation between plasma L-OHP concentration and the time of onset of neuropathy (24).

Acute cold allodynia usually occurs at the onset of the first 
regimen cycle in most patients. The mechanism of acute cold allodynia was reported by Zhao et al.; according to them, in cold allodynia, sodium calcium channels are damaged, thereby resulting in a decrease in the influx of calcium (27). This means that the acute cold allodynia is related to not the L-OHP metabolites but the intact L-OHP. The fraction of intact L-OHP was related to the onset and severity of acute cold allodynia. Therefore, we investigated whether the determination of intact L-OHP was associated with various pharmacokinetic parameters. Here we report the findings of an in vivo study of plasma L-OHP concentration, and its relative accumulation following repeated administrations of L-OHP. Acute cold allodynia was observed after just two days of treatment with 3,5 , or $8 \mathrm{mg} / \mathrm{kg}$ of L-OHP in rats. The withdrawal response was significantly increased with respect to the control group. A positive correlation $\left(\mathrm{R}^{2}\right.$ $=0.9816)$ was observed between the number of withdrawal responses and the AUC of the intact L-OHP. This result suggests that monitoring of intact L-OHP in the plasma of patients may help to prevent the onset of acute cold allodynia. Unlike the results for L-OHP, when we analyzed plasma platinum levels, we did not observe a good correlation with acute cold allodynia. This suggests that the response observed here is related to accumulation of intact L-OHP and not that of its metabolites.

\section{Conclusions}

In conclusion, as cold allodynia occurs during the early phases of chemotherapy, and its onset is linked to L-OHP concentration, determination of the concentration of intact L-OHP may be useful for identification of acute neuropathy, thereby allowing for early dosage adjustment and aiding the improvement of drug regimen compliance.

\section{Acknowledgments}

We would like to thank Professor Toshiyuki Sakaeda for his invaluable help and support during the writing of this article. This study would not have been possible without his guidance.

Funding: This study was supported by a Grant-in-Aid for Scientific Research (C) from JSPS KAKENHI, Grant Number 15K08085.

\section{Footnote}

Reporting Checklist: The authors have completed the
ARRIVE reporting checklist. Available at http://dx.doi. org/10.21037/apm-20-542

Data Sharing Statement: Available at http://dx.doi. org/10.21037/apm-20-542

Conflicts of Interest: All authors have completed the ICMJE uniform disclosure form (available at http://dx.doi. org/10.21037/apm-20-542). The authors have no conflicts of interest to declare.

Ethical Statement: The authors are accountable for all aspects of the work in ensuring that questions related to the accuracy or integrity of any part of the work are appropriately investigated and resolved. All animal protocols were approved by the Institutional Animal care and Use Committee (No. PKPD-16-001) and were conducted in accordance with the Guidelines for Animal Experimentation, Kyoto Pharmaceutical University.

Open Access Statement: This is an Open Access article distributed in accordance with the Creative Commons Attribution-NonCommercial-NoDerivs 4.0 International License (CC BY-NC-ND 4.0), which permits the noncommercial replication and distribution of the article with the strict proviso that no changes or edits are made and the original work is properly cited (including links to both the formal publication through the relevant DOI and the license). See: https://creativecommons.org/licenses/by-nc-nd/4.0/.

\section{References}

1. André T, Boni C, Navarro M, et al. Improved overall survival with oxaliplatin, fluorouracil, and leucovorin as adjuvant treatment in stage II or III colon cancer in the MOSAIC trial. J Clin Oncol 2009;27:3109-16.

2. de Gramont A, Figer A, Seymour M, et al. Leucovorin and fluorouracil with or without oxaliplatin as first-line treatment in advanced colorectal cancer. J Clin Oncol 2000;18:2938-47.

3. Haller DG, Tabernero J, Maroun J, et al. Capecitabine plus oxaliplatin compared with fluorouracil and folinic acid as adjuvant therapy for stage III colon cancer. J Clin Oncol 2011;29:1465-71.

4. Kuebler JP, Wieand HS, O'Connell MJ, et al. Oxaliplatin combined with weekly bolus fluorouracil and leucovorin as surgical adjuvant chemotherapy for stage II and III colon cancer: results from NSABP C-07. J Clin Oncol 
2007;25:2198-204.

5. Rothenberg ML, Oza AM, Bigelow RH, et al. Superiority of oxaliplatin and fluorouracil-leucovorin compared with either therapy alone in patients with progressive colorectal cancer after irinotecan and fluorouracil-leucovorin: interim results of a phase III trial. J Clin Oncol 2003;21:2059-69.

6. Grothey A. Oxaliplatin-safety profile: neurotoxicity. Semin Oncol 2003;30:5-13.

7. Adelsberger H, Quasthoff S, Grosskreutz J, et al. The chemotherapeutic oxaliplatin alters voltage-gated $\mathrm{Na}(+)$ channel kinetics on rat sensory neurons. Eur J Pharmacol 2000;406:25-32.

8. Grolleau F, Gamelin L, Boisdron-Celle M, et al. A possible explanation for a neurotoxic effect of the anticancer agent oxaliplatin on neuronal voltage-gated sodium channels. J Neurophysiol 2001;85:2293-7.

9. Park SB, Goldstein D, Lin CS, et al. Acute abnormalities of sensory nerve function associated with oxaliplatininduced neurotoxicity. J Clin Oncol 2009;27:1243-9.

10. Webster RG, Brain KL, Wilson RH, et al. Oxaliplatin induces hyperexcitability at motor and autonomic neuromuscular junctions through effects on voltage-gated sodium channels. Br J Pharmacol 2005;146:1027-39.

11. Jamieson SM, Liu J, Connor B, et al. Oxaliplatin causes selective atrophy of a subpopulation of dorsal root ganglion neurons without inducing cell loss. Cancer Chemother Pharmacol 2005;56:391-9.

12. Screnci D, McKeage MJ, Galettis P, et al. Relationships between hydrophobicity, reactivity, accumulation and peripheral nerve toxicity of a series of platinum drugs. Br J Cancer 2000;82:966-72.

13. Yan F, Liu JJ, Ip V, et al. Role of platinum DNA damageinduced transcriptional inhibition in chemotherapyinduced neuronal atrophy and peripheral neurotoxicity. J Neurochem 2015;135:1099-112.

14. Kawashiri T, Egashira N, Kurobe K, et al. L type $\mathrm{Ca}^{2}+$ channel blockers prevent oxaliplatin-induced cold hyperalgesia and TRPM8 overexpression in rats. Mol Pain 2012;8:7.

15. Shirahama $M$, Ushio $S$, Egashira $N$, et al. Inhibition of $\mathrm{Ca} 2+$ /calmodulin-dependent protein kinase II reverses oxaliplatin-induced mechanical allodynia in rats. Mol Pain 2012;8:26.

16. Jerremalm E, Wallin I, Ehrsson H. New insights into the biotransformation and pharmacokinetics of oxaliplatin. J Pharm Sci 2009;98:3879-85.

17. Jerremalm E, Hedeland M, Wallin I, et al. Oxaliplatin degradation in the presence of chloride: identification and cytotoxicity of the monochloro monooxalato complex. Pharm Res 2004;21:891-4.

18. Han CH, Khwaounjoo P, Hill AG, et al. Predicting effects on oxaliplatin clearance: in vitro, kinetic and clinical studies of calcium- and magnesium-mediated oxaliplatin degradation. Sci Rep 2017;7:4073.

19. Ito H, Yamaguchi H, Fujikawa A, et al. A full validated hydrophilic interaction liquid chromatography-tandem mass spectrometric method for the quantification of oxaliplatin in human plasma ultrafiltrates. J Pharm Biomed Anal 2012;71:99-103.

20. Onose J, Imai T, Hasumura M, et al. Rapid induction of colorectal tumors in rats initiated with 1,2-dimethylhydrazine followed by dextran sodium sulfate treatment. Cancer Lett 2003;198:145-52.

21. Ito Y, Kobuchi S, Takesada W, et al. Assessment of Oxaliplatin-induced Chronic Neuropathy and Anticancer Efficacy Through Pharmacokinetic and Toxicodynamic Evaluation of a Rat Model of Colorectal Cancer. Anticancer Res 2019;39:4207-13.

22. Flatters SJ, Bennett GJ. Ethosuximide reverses paclitaxeland vincristine-induced painful peripheral neuropathy. Pain 2004;109:150-61.

23. Johnsson A, Björk H, Schütz A, et al. Sample handling for determination of free platinum in blood after cisplatin exposure. Cancer Chemother Pharmacol 1998;41:248-51.

24. Ito Y, Kobuchi S, Shimizu R, et al. Pharmacokinetic and toxicodynamic evaluation of oxaliplatin-induced neuropathy and hematological toxicity in rats. Cancer Chemother Pharmacol 2018;81:155-61.

25. Tournigand C, Cervantes A, Figer A, et al. OPTIMOX1: a randomized study of FOLFOX4 or FOLFOX7 with oxaliplatin in a stop-and-Go fashion in advanced colorectal cancer--a GERCOR study. J Clin Oncol 2006;24:394-400.

26. Grothey A, Nikcevich DA, Sloan JA, et al. Intravenous calcium and magnesium for oxaliplatin-induced sensory neurotoxicity in adjuvant colon cancer: NCCTG N04C7. J Clin Oncol 2011;29:421-7.

27. Zhao M, Isami K, Nakamura S, et al. Acute cold hypersensitivity characteristically induced by oxaliplatin is caused by the enhanced responsiveness of TRPA1 in mice. Mol Pain 2012;8:55.

Cite this article as: Ito Y, Kobuchi S, Takeda M, Morimoto M. Effect of intact oxaliplatin in plasma on a cold allodynia after multiple administrations in colorectal cancer model rats. Ann Palliat Med 2020;9(5):3000-3006. doi: 10.21037/apm-20-542 